\title{
Diversidade de bactérias ambientais e de pacientes no Hospital Geral de Palmas-TO
}

Anderson Barbosa BAPTISTA ${ }^{[1, *]}$; João Marcos Monteiro RAMOS [2]; Rodolfo Rezende das NEVES [2]; Douglas Ferreira de SOUZA ${ }^{[3]}$ e Raphael Sanzio PIMENTA ${ }^{[1]}$

${ }^{[1]}$ Docente do curso de Medicina da Universidade Federal do Tocantins, Campus Palmas. Avenida NS 15, 109 Norte - Plano Diretor Norte, 77001-090. Palmas-TO, Brasil. Email: pimentars@uft.edu.br

${ }^{[2]}$ Acadêmico do curso de Medicina da Universidade Federal do Tocantins, Campus Palmas. Avenida NS 15, 109 Norte - Plano Diretor Norte, 77001-090. Palmas-TO, Brasil. E-mail: jotamarcos_1992@ hotmail.com, rodolfo-rezende@ hotmail.com

${ }^{[3]}$ Biomédico do Laboratório CMD do Hospital Geral de Palmas. 201 Sul Avenida NS A, QD-43A, LT-1 Plano Diretor Sul, $77015-$ 205. Palmas-TO, Brasil. Email: douglaspso@ hotmail.com

INFORMAÇÕES

Recebido em: 05/09/2015

Aceito em: 20/11/2015

Publicado em: 23/12/2015

Document Object Identifier

$10.18067 /$ jbfs.v2i4.63

Termos de indexação:

Resistência a Fármacos,

Medidas profiláticas

Serratia marcescens

Klebsiella pneumoniae

*Autor para correspondência

$\underline{\text { andersonbb@uft.edu.br }}$

\section{RESUMO}

Patógenos resistentes representam um grande desafio para os hospitais no que se referem aos ao tratamento e as medidas profiláticas. Uma vez no ambiente cepas Gram negativas podem adquirir genes de resistência do solo, da água e dos efluentes hospitalares e assim transmiti-los a outros gêneros. Objetivou-se neste estudo quantificar e qualificar as cepas bacterianas presentes nos pacientes com suspeita de infecção hospitalar e no ambiente em que se encontram internados do Hospital Geral de Palmas. Foram encontradas 14 espécies bacterianas diferentes de 141 amostras, sendo 123 de pacientes e 18 do ambiente hospitalar. Nas amostras dos pacientes destacaram-se a Klebsiella pneumoniae (21,9\%), Acinetobacter baumannii (13,8\%), Pseudomonas aeruginosa (12,2\%), Escherichia coli (11,4\%), Serratia marcescens (10, $6 \%)$ e Staphylococcus aureus (8,9\%), dessas observou-se que 80 cepas eram multirresistentes aos antimicrobianos, prevalecendo a Klebsiella pneumoniae $(26,3 \%) \mathrm{e}$ a Acinetobacter baumannii (20,0\%). As amostras de Aspirado traqueal apresentaram um maior número de cepas multirresistentes. No ambiente prevaleceram a Serratia marcescens, Staphylococcus aureus e Staphylococcus sp. ambas com 22,2\%, coletadas de diferentes locais e materiais da UTI como tubo traqueal, Ambu, colchão e monitores. Estes resultados apontam a necessidade de implantação de estratégias administrativas e educativas para reduzir a disseminação de cepas multirresistentes.

\section{Diversity of environmental and patients bacteria in the Hospital Geral de Palmas-TO.}

ABSTRACT- Resistant pathogens are an important challenge to hospitals, especially to stablish the correct treatment and prophylactic measures. Once in the environmental Gram-negative strains can acquire resistance genes from the soil, water and hospital effluent and so transmit it to other genus. The objective of this study was to quantify and to qualify the bacterial strains present in patients with suspected of nosocomial infection and the environment in which they are admitted in the Palmas General Hospital. 14 different bacterial species from 141 samples, being 123 from patients and 18 from hospital environment. In patient samples highlights were Klebsiella pneumoniae (21.9\%), Acinetobacter baumannii (13.82\%), Pseudomonas aeruginosa (12.2\%), Escherichia coli (11.4\%), Serratia marcescens $(10.6 \%)$ and Staphylococcus aureus $(9.0 \%)$, From this total, 80 strains were multiresistant to antibiotics, whichever Klebsiella pneumoniae (26.3\%) and A. baumannii (20.0\%). The tracheal aspirate samples showed a greater number of multidrug-resistant strains. The environment prevailed Serratia marcescens, Staphylococcus aureus and Staphylococcus sp. both with $22.2 \%$, collected from different locations and ICU materials such as tracheal tube, Ambu, mattress and monitors. Administrative and educational strategies are necessary to reduce the spread of multi-resistant germs.

Index terms: Drug resistance, Prophylactic measures, Serratia marcescens, Klebsiella pneumoniae

(i) (2) Copyright: (C) 2015 JBFS all rights. This is an open-access article distributed under the terms of the Creative Commons Attribution License, which permits unrestricted use, distribution, and reproduction in any medium, provided the original author and source are credited.

Financiamento: Os autores reportam que não houve suporte e auxílio financeiro

Conflito de interesse: Os autores declaram que não há conflito de interesse.

Comitê de Ética em Pesquisa com seres humanos: Processo 247/2013 UFT - Aprovado em 27/04/2014.

Como referir esse documento (ABNT):

BAPTISTA, A. B.; RAMOS, J. M. M.; NEVES, R. R.; SOUZA, D. F.; PIMENTA, R. S. Diversidade de bactérias ambientais e de pacientes no Hospital Geral de Palmas-TO. Journal of Bioenergy and Food Science, Macapá, v.2, n.4, p.160-164, out./dez., 2015. http://dx.doi.org/10.18067/jbfs.v2i4.63 


\section{INTRODUÇÃO}

O ambiente hospitalar envolve a exposição dos profissionais de saúde e demais trabalhadores a uma diversidade de riscos, especialmente os biológicos, sendo o mesmo um local propício a diversos tipos de infecções devido à circulação, aumento e a virulência de agentes patogênicos presentes [1]. Infecções nosocomiais são frequentemente observadas em Unidades de Tratamento Intensivo e condutas profiláticas para evitar a multirresistência bacteriana [2, 3].

Várias espécies bacterianas são capazes de disseminar-se no ambiente hospitalar, no grupo das enterobactérias os gêneros mais frequentes isolados em amostras biológicas são Escherichia coli, Klebsiella ssp., Serratia ssp., Proteus ssp., Enterobacter ssp., Providencia ssp., Morganella ssp., Salmonela sp. e Shigella sp. [4]. No centro de terapia intensiva as bactérias mais frequentes são Escherichia coli, Staphylococcus aureus e Pseudomonas aeruginosa [5, 6].

Vêm se registrando um aumento nos casos de multirresistência bacteriana, devido à pressão seletiva causada pelo uso inadequado de antimicrobianos. Existe uma associação desse grupo às infecções hospitalares e o aumento das infecções, com maior risco para os pacientes mais debilitados $[7,8]$.

A contaminação de superfícies é comum em áreas inanimadas e equipamentos. Nos EUA identificaram frequente contaminação de superfície por Enterococcus resistentes à vancomicina (VRE) e Staphylococcus aureus resistente à meticilina (MRSA). Ainda não está claro como os microorganismos que sobrevivem no ambiente são transmitidos para os pacientes o quadro clínico, a má desinfecção de material do leito, a limpeza ineficiente, estrutura física, má assepsia das mãos podem ser fatores determinantes [9].

O objetivo desse estudo foi isolar bactérias de pacientes com suspeita de infecção Hospitalar e do ambiente dos leitos do Hospital Geral de Palmas, para conhecer a diversidade e a epidemiologia.

\section{MATERIAIS E MÉTODOS}

\section{Trata-se de um estudo descritivo exploratório, prospectivo com abordagem quantitativa e qualitativa.}

Foram coletadas 123 amostras dos pacientes internados em vários ambientes e 18 amostras ambientais de utensílios, mobiliários e equipamentos nos leitos equivalentes a cada paciente objeto desse estudo, do Hospital Geral de
Palmas (HGP), estado do Tocantins, no período de janeiro a abril de 2015 .

Os ambientes escolhidos para a pesquisa foram a Unidades de Tratamento Intensivo e Unidade de Cuidados Intensivos, para as amostras ambientais e dos pacientes foram incluídas as áreas de internação.

Os artigos de superfície foram escolhidos aleatoriamente, nos leitos e nas salas, com a técnica de fricção de $s w a b$ estéril, utilizando apenas uma coleta em cada artigo, e imediatamente colocados em tubo com caldo Tioglicolato. As culturas foram levadas ao laboratório de Microbiologia da UFT e incubadas em estufa a $37^{\circ} \mathrm{C}$, por 24 horas. $\mathrm{Na}$ sequência foram semeadas nos meios de cultura Ágar MacConkey, Ágar Mueller Hinton, Ágar Manitol e Ágar Verde Brilhante, após crescimento, as colônias foram submetidas a provas bioquímicas específicas para enterobactérias e bactérias Gram positivas.

As amostras dos pacientes foram coletadas pelo laboratório CMD do HGP o qual reportou as espécies isoladas e o perfil de resistência bacteriana, utilizando meios Ágar Sangue de Carneiro, Ágar Mac Conkey, para série bioquímica de enterobactérias utilizou-se o Sistema Bactray II e III (Laborclin). Os antibiogramas foram analisados pela técnica de Kirby Bauer, de difusão em disco, utilizando antimicrobianos preconizados pela ANVISA e padronizados ao Hospital. Todas as cepas foram novamente cultivadas no laboratório de microbiologia da UFT e estocadas em caldo tioglicolato acrescido deglicerol $15 \%$ e mantidos em a $-80^{\circ} \mathrm{C}$. Projeto autorizado pelo Comitê de Ética em Pesquisa da UFT, processo 247/2013.

\section{RESULTADOS E DISCUSSÃO}

Foram identificadas 14 espécies bacterianas em 141 amostras totais (Tabela 1). Nas amostras de origem clínica destacaram-se a Klebsiella pneumoniae (21,9\%), Acinetobacter baumannii (13,8\%), Pseudomonas aeruginosa (12,2\%), Escherichia coli (11,4\%), Serratia marcescens $(10,6 \%)$ e Staphylococcus aureus $(8,9 \%)$, dessas observaram-se que 80 cepas eram multirresistentes aos antimicrobianos, especialmente à classe de Beta Lactâmicos, prevalecendo a Klebsiella pneumoniae (26,2\%) e a Acinetobacter baumannii (20\%). Foi considerada multirresistência para as bactérias que eram resistentes a mais de uma classe de antimicrobianos.

Entre os exames realizados a urocultura que mais apresentou crescimento bacteriano, um total de 33 , no qual $61 \%$ eram multirresistentes e o aspirado traqueal em 30 amostras apresentando $73 \%$ de multirresistência, coletados de vários ambientes do 
hospital entre pacientes com diferentes condições de internação.

Tabela 1. Diversidade de espécies isoladas de pacientes e ambientais do Hospital Geral de Palmas-TO.

\begin{tabular}{|c|c|c|}
\hline Espécie isolada & Frequência & $\begin{array}{l}\text { Frequência } \\
(\%)\end{array}$ \\
\hline Acinetobacter baumannii & 18 & 12,8 \\
\hline Enterobacter cloacae & 3 & 2,1 \\
\hline Enterococcus sp & 7 & 4,9 \\
\hline Escherichia coli & 14 & 9,9 \\
\hline Klebsiella ozaenae & 1 & 0,7 \\
\hline Klebsiella pneumoniae & 29 & 20,7 \\
\hline Morganella morganii & 1 & 0,7 \\
\hline Proteus mirabilis & 3 & 2,1 \\
\hline Proteus vulgaris & 1 & 0,7 \\
\hline Pseudomonas aeruginosa & 23 & 16,3 \\
\hline Serratia marcescens & 17 & 12,0 \\
\hline Staphylococcus aureus & 15 & 10,7 \\
\hline Staphylococcus sp & 4 & 2,9 \\
\hline $\begin{array}{l}\text { Stenotrophomonas } \\
\text { maltophilia }\end{array}$ & 5 & 3,5 \\
\hline Total & 141 & 100 \\
\hline
\end{tabular}

Um estudo realizado em Terezina, em um Hospital público, encontraram dados que corroboram com este estudo, pois encontraram o maior número de infecções relacionadas a UTI em Topografias respiratória $60,12 \%$, sistêmica $17,8 \%$ e urinária 16,24. As bactérias que mais prevaleceram nas UTI foram: Klebsiella pneumoniae $(33,7 \%)$ e Pseudomonas sp. (24,0\%) [10].

Larrinaga, et al. [11] estudaram as infecções por Klebsiella pneumoniae e Acinetobacter baumannii em Hospitais Infantis de Cuba, para tanto encontraram maior isolamento da primeira em Neonatologia $(33 \%)$ e na Unidade de Cuidados Intensivos Pediátricos (32\%) para a segunda. Dentro do gênero Klebsiella encontraram $96 \%$ de $K$. pneumoniae, $3 \%$ de $K$. oxytoca e $1 \%$ K. ozaenae. Para Acinetobacter foram isoladas $90 \%$ de $A$. baumannii, 4\% A. haemolyticus e A. iwoffi e $2 \%$ A. johnsonii, causando infecções em crianças internadas. Dados semelhantes a este estudo que observou maior prevalência de bactérias multirresistentes Klebsiella pneumoniae e a Serratia marcescens na Unidade de Tratamento Intensivo Adulto.No ambiente prevaleceram Serratia marcescens, Staphylococcus aureus e Staphylococcus sp ambas com $22,2 \%$, coletadas em equipamentos e móveis da UTI e UCI, sendo isolada apenas uma cepa patogênica em cada amostra (Tabela 2).

Tabela 2. Artigos de superfície coletados na UTI e na UCI e espécie bacteriana isolada no Hospital Geral de Palmas-TO.

\begin{tabular}{|c|c|}
\hline $\begin{array}{c}\text { Artigo de superfície e local do } \\
\text { Hospital }\end{array}$ & Espécie bacteriana \\
\hline $\begin{array}{l}\text { Tubo traqueal desconectado do } \\
\text { paciente/ UCI }\end{array}$ & Serratia marcescens \\
\hline $\begin{array}{l}\text { Colchão em torno do paciente/ } \\
\text { UTI }\end{array}$ & Stahphylococcus sp \\
\hline $\begin{array}{l}\text { Mangueira da sonda } \\
\text { abdominal/ UTI }\end{array}$ & Serratia marcescens \\
\hline $\begin{array}{l}\text { Ambu em cima da bancada do } \\
\text { leito/ UTI }\end{array}$ & Serratia marcescens \\
\hline $\begin{array}{l}\text { Tubo de respiração } \\
\text { desconectado do paciente, por } \\
\text { dentro/ UTI }\end{array}$ & Klebsiella penumoniae \\
\hline Botões do monitor/ UTI & Staphylococcus aureus \\
\hline Pia de um leito/ UTI & Staphylococcus sp \\
\hline
\end{tabular}
paciente de um leito/ UTI

Staphylococcus aureus

Botões do monitor cardíaco/ UTI

Staphylococcus sp

Mangueira do Tubo de respiração, parte externa/ UTI

Klebsiella penumoniae

Mangueira da sonda

nasogástrica/ UCI

Proteus mirabilis

Colchão em contato com escaras/ UCI

Staphylococcus aureus

Cama e lençóis/ UCI

Staphylococcus aureus

Sonda nasogástrica/ UTI

Staphylococcus sp

Bomba de infusão/ UTI

Staphylococcus sp

Bancada da enfermaria/ UTI

Acinetobacter baumanni

Mangueira do catéter nasal/ UTI

Stenotrofomonas maltophilia

Tubo traqueal por dentro/ UCI Serratia marcescens

Renner e Carvalho [6] analisaram 45 superfícies com crescimento bacteriano em $84,4 \%$ das amostras. Destas $42 \%$ era Staphylococcus epidermidis, 37\% S. aureus, 3\% de Enterococcus, $3 \%$ de K. pneumoniae e $15 \%$ de Bacillus spp. O 
leito 6 encontraram na mesa, na cama, no estetoscópio, no dispensador de soro contaminação por Staphylococcus aureus e no respirador mecânico Staphylococcus epidermidis. Há destaque para o crescimento em superfícies também evidenciado neste estudo, porém com prevalência de Serratia marcescens.

Ferreira et al., [13] coletaram amostras de colchão de um Hospital escola, antes e depois da lavagem e encontraram contaminação de Staphylococcus aureus em 55,4\% antes e 44,6\% depois, e entre os 15 colchões analisados, de 5 foram isoladas Staphylococcus aureus meticilina resistente. Esse microrganismo é considerado o principal agente causador de infecções adquiridas na comunidade e no ambiente hospitalar. A contaminação nos colchões se dá pelo contato da pele e/ou fluidos dos pacientes, esse microorganismo, segundo evidências, pode sobreviver por muito tempo no ambiente inanimado.

As mãos dos profissionais de saúde e o ambiente hospitalar podem ser uma fonte de contaminação de bactérias como o Staphylococcus aureus, Pseudomonas sp., Acinetobacter spp., Enterococcus spp. e o Clostridium difficile. Quando se compara a outras bactérias a Acinetobacter baumannii tem maior transmissibilidade através do ambiente e superfícies inanimadas [13].

Um estudo feito em Unidades de Terapia Intensiva em um Hospital em Recife, amostras coletadas de superfícies, encontraram $24,4 \%$ de Acinetobacter baumannii multirresistente, com maior contaminação nos respiradores e bombas de infusão, respectivamente [14] (SALES et al., 2014).

\section{CONCLUSÃO}

A diversidade de bactérias isoladas no Hospital Geral de Palmas e a multirresistência tem grande importância para avaliação das questões profiláticas como a limpeza do ambiente, assepsia de mãos, isolamento de alguns pacientes, uso correto de avental e luvas. Entre outras medidas, para evitar contaminações cruzadas e procedimentos inadequados.

A UTI é um local que merece atenção, pois é bastante vulnerável às disseminações de patógenos pelo aspecto físico e paciente de cuidados intensivos que possuem maior risco de infecções. Especialmente quando são isoladas cepas multidroga resistentes, pois a terapêutica fica restrita, com poucas alternativas. As superfícies que estão próximas ou em contato com os pacientes precisam de maior cuidado com a assepsiam, utilizando-se de técnicas e antissépticos que minimizem as contaminações.

O uso racional de antimicrobianos, atividades educativas, acompanhamento de culturas, perfis de resistência e vigilância de cepas isoladas dos hospitais contribuem para diminuir a presença de diversos grupos de bactérias, a multirresistência e as contaminações.

\section{AGRADECIMENTOS}

Laboratório CMD do HGP e Senhora Carolina do Núcleo de Ensino do HGP, Palmas-TO.

\section{CONTRIBUIÇÃO DOS AUTORES}

1. Coleta das amostras no Hospital Geral de Palmas. Identificação das bactérias e testes de resistência:

Anderson Barbosa Baptista

Douglas Ferreira de Souza

João Marcos Monteiro Ramos

Rodolfo Rezende das Neves.

2. Confecção do manuscrito, tratamento dos dados e orientação geral:

Anderson Barbosa Baptista

Dr.Raphael Sanzio Pimenta

www.pnas.org/lookup/suppl/doi:10.1073/pnas.13080 62111/-/DCSupplemental

[3].AYCAN, I.O.; CELEN, M.K.; YILMAZ, A.; ALMAZ, M.S.; DAL, T.; CELIK Y.; BOLAT, E. Colonização bacteriana por causa do aumento da carga de trabalho da equipe de enfermagem em unidade de terapia intensiva. Revista Brasileira Anestesiologia, v.65, n.3, p.180-185, 2015. http://dx.doi.org/10.1016/j.bjan.2014.05.004

[4]. OLIVEIRA, M.C. Enterobacteriaceae resistentes às cefalosporinas de terceira geração isoladas em amostras obtidas nas primeiras 48 horas de internação em um hospital universitário: frequência, fatores de 
risco e impactos na evolução clínica. 2013. 81p. Dissertação (Mestrado) - Universidade Federal de Minas Gerais, Belo Horizonte

[5].JACOBY, T.S. Associação entre consumo de antimicrobianos e multirresistência bacteriana em centro de terapia intensiva de Hospital Universitário Brasileiro, 2004-2006. 2008. 108p. Dissertação (Mestrado em Ciências Médicas), Universidade Federal do Rio Grande do Sul, PORTO ALEGRE

[6].RENNER， J.D.P. \& CARVALHO E.D. Microorganismos isolados de superfícies da UTI adulta em um hospital do Vale do Rio Pardo - RS. Revista de Epidemiologia e Controle de Infecção, v.3, n.2, p.40-44,2013. http://dx.doi.org/10.17058/reci.v3i2.3290

[7].ANDRADE, D.; LEOPOLDO, V.C.; HAAS, V.J. Ocorrência de bactérias multiresistentes em um centro de Terapia Intensiva de Hospital brasileiro de emergências. Revista brasileira terapia intensiva, v.18, n.1, p.27-33, 2006. http://dx.doi.org/10.1590/S0103507X2006000100006

[8].ALVES, I.A.; OLIVEIRA, M.E.A.; OLIVEIRA, V.A.; ROCHA, D.B.; GOMES, D.A.V. Isolamento de bactérias multirresistentes coletadas dos espéculos utilizados em coletas de exames citopatológicos em postos de saúde da rede pública de um município de médio porte no Rio Grande do Sul. Revista de Iniciação Científica da ULBRA, v. 10: p. 413-423, 2012.

[9]. OLIVEIRA, A.C.; DAMASCENO, QS. Superfícies do ambiente hospitalar como possíveis reservatórios de bactérias resistentes: uma revisão. Revista da escola de enfermagem USP, São Paulo, v. 44, n. 4, p.1118-1123, 2010. http://dx.doi.org/10.1590/S0080$\underline{62342010000400038}$

[10]. MOURA, M.E.B.; CAMPELO, S.M.A.; BRITO, F.C.P.; BATISTA, ODINÉA M.A.;
ARAÚJO, T.M.E.; OLIVEIRA, ADÉLIA DS. Infecção hospitalar: estudo de prevalência em um hospital público de ensino. Revista Brasileira Enfermagem, v.60, n.4, p.416-421, 2007. http://dx.doi.org/10.1590/S003471672007000400011

[11]. LARRINAGA, Y.S.; CARTAYA, Y.C.; FARIA, Y.C.P.; NOVOA, L.D.; GIRO, SG; KOBAYASHI, N.; PÉREZ II D.Q. Infecciones por los géneros Klebsiella y Acinetobacter en hospitales pediátricos cubanos y resistencia antibiótica. Revista Cubana de Medicina Tropical, v.66, n.3, p.400-414, 2014

[12]. FERREIRA, A.M.; ANDRADE, D.A.; MARGARETE T.G.C.; KEITH, C.; RIGOTTI, M.A. Colchões do tipo caixa de ovo: um reservatório de Staphylococcus aureus resistente à meticilina? Revista da Escola de Enfermagem da USP, v.45, n.1, p.161-166, 2011. http://dx.doi.org/10.1590/S0080$\underline{62342011000100022}$

[13]. KUPLICH, N.M.; GASTAL, S.L.; DEUTSCHENDORF, C.; JACOBY, T.S.; LOVATTO, C.G.; KONKEWICZ, L.R.; PIRES, M.R.; NAGEL, F.; MACHADO, D.P.; AQUINO, V.R.; SANTOS, R.P. Política de prevenção da disseminação de germes multirresistentes no hospital de clínicas de porto alegre. Revista Hospital de Clínicas de Porto Alegre, v.31, n.1, p.80-89, 2011.

[14]. SALES, V.M.; OLIVEIRA, E.; CÉLIA, R.; GONÇALVES, F.R.; MELO, C.C. Análise microbiológica de superfícies inanimadas de uma Unidade de Terapia Intensiva e a segurança do paciente. Revista de Enfermagem, v.4, n.3 p. 45-53, 2014. http://dx.doi.org/10.12707/RIII1293 